\title{
An effective procedure for changing food preferences in 5-7-year-old children
}

\author{
BY PAULINE J. HORNE, C. FERGUS LOWE, \\ PAUL F. J. FLEMING AND ALAN J. DOWEY \\ School of Psychology, University of Wales, Bangor, Gwynedd LL57 2DG
}

'What people can eat is biologically determined; what they do eat is quite another matter'

(Levins \& Lewontin, 1985, p. 262)

In many cultures despite there being a vast array of possible foods from which people could choose their diet, only a relatively small selection is consumed. Why this should be the case and how people might be influenced to eat more varied and healthy diets is the main focus of the present paper.

It is widely acknowledged that many factors determine the acquisition and alteration of food preferences, in humans. Two broad domains of influence, however, may be identified, namely, biological and socio-cultural. Biological factors include apparently innate preferences for sweet-tasting substances (Desor et al. 1973; Crook, 1978), genetic contributions to taste sensitivity (Fischer et al. 1961; Glanville \& Kaplan, 1965; Jefferson \& Erdman, 1970; Hall et al. 1975; Davis, 1978) and genetic influences on metabolic processes (Simoons, 1969, 1970). It is argued, however, that most individual differences in food preferences are attributable to socio-cultural rather than biological influences (Greene et al. 1975; Rozin, 1982) and it is the former we shall concentrate on here.

Cultural practices in relation to food are based on the accumulated experiences of millions of individuals across time. Individuals benefit from, and make use of, this communal experience eliminating the need for their own trial-and-error learning with every new potential food item. In its broadest sense, cultural practice defines which substances are 'foods' and which are 'non-foods'. Thus, people from different ethnic origins have different views about acceptable food substances. For example, South American Indians eat monkeys, grubs, bees, and headlice, Australian Aborigines relish insects, and some Sri Lankan tribes eat rotted wood (Fieldhouse, 1991). None of these foods feature in the European diet. Similarly, cultural practice indicates acceptable combinations of foods (e.g. in Western culture, not cake with steak), appropriate times for the consumption of food items (e.g. cereal for breakfast not lunch) and methods of preparation. How is such cultural influence achieved?

\section{OUR PERSPECTIVE}

Our theoretical approach draws on contemporary accounts of conditioning and learning processes (Lowe, 1983; Catania, 1992) in combination with the work of L. S. Vygotsky in 1934 (Vygotsky, 1987) and G. H. Mead (1934) on the socio-cultural determinants of language and its role in regulating human behaviour (see also Diaz, 1992). According to this view, while we should expect classical and operant conditioning processes, as observed in a range of animal species, to influence much of human behaviour, humans are also subject to particular social influences which do not affect the behaviour of other animal species, the most unique and important being language (Lowe, 1979, 1983, 1989; 
Lowe et al. 1987). A child is born into a particular culture and learns to name objects and events. These names become, in turn, the child's tools of thinking allowing him (or her) to conceptualize the world and talk about his (or her) relationship to it. Moreover, because language is used by the culture as the means of communication, the way that children name and categorize their environment necessarily reflects the practices of their particular culture. Although initially these names are taught to the child by the culture, with continued interaction and growing proficiency in language children come to create their own labels and categories. However names are acquired, they come to mediate and govern the child's entire behavioural repertoire; responses to food, of course, form an important subset of this repertoire.

Thus, a child's food preferences may be established directly by operant or classical conditioning effects or by observation of others' (e.g. family, friends, etc.) food-related behaviours. In the course of acquiring language, however, the impact of environmental stimuli, including foods, is transformed as the child learns to respond not directly to particulars but to named classes of objects and events (e.g. 'tomatoes', 'vegetables', 'cheese') and to frame complex rules to govern his (or her) behaviour. For example, having heard a friend express distaste for tomatoes (which may not even be present at the time) a child may say thereafter that he (or she) too 'hates tomatoes' and may refuse to eat them. Such verbal classifications and rules can profoundly alter future eating behaviour, both in the case of foods already encountered as well as in the case of new foods that may be perceptually similar to, or categorized in the same way as, those previously encountered by the child. We see it, therefore, as essential to understand how people's verbal constructions of foods are established and how they interact with basic conditioning processes and biological determinants.

Our general approach is supported by evidence from a range of research sources, all of which are concerned with people's, particularly children's, use of language to conceptualize their environment and to formulate rules for behaviour. These include: (1) developmental analyses of children's learning and how it is transformed by the acquisition of linguistic skills (e.g. Lowe et al. 1983; Bentall et al. 1985; Lowe \& Horne, 1985; Bentall \& Lowe, 1987; Catania et al. 1989, 1990; Hayes, 1989; Dugdale \& Lowe, 1990), (2) approaches in clinical psychology which have employed verbal and cognitive procedures to change behaviour (e.g. Lowe \& Higson, 1983; Woods \& Lowe, 1986; Lowe et al. 1987; Chadwick \& Lowe, 1990; Lowe \& Chadwick, 1990; Meichenbaum, 1990; Whitman, 1990), (3) experimental investigations which employ 'metacognitive' training to instruct children in techniques of self-monitoring and self-regulation (Lowe \& Higson, 1981; Brown \& Campione, 1984; Pressley et al. 1987), (4) studies of 'correspondence' training in which verbal commitments to carry out a particular behaviour are rewarded only if followed by the announced behaviour; for example, a child's statement that 'Tomorrow I will brush my teeth' would qualify for a reward only if followed by the toothbrushing (Baer et al. 1985; Deacon \& Konarski, 1987). To date, little research on children's food preferences has incorporated the findings from these different research approaches, whether in analysing the development of children's eating patterns or in changing them.

Before describing an empirical study which is based on the theoretical perspectives outlined previously, we briefly review the literature dealing with some of the variables central to our research programme, namely, the effects of (1) repeated exposure, (2) modelling, and (3) rewards. 


\section{EXPOSURE}

Zajonc (1968) has proposed that repeated exposure to any of a range of visual and auditory stimuli may lead to increased liking for the stimuli in question; a phenomenon referred to as the 'mere exposure' effect. Determining whether this occurs in foodpreference acquisition is complex in that in most real-life settings food exposure almost invariably involves a compound of sight, olfaction and taste. Pliner (1982), attempted to assess the impact of taste exposure on preferences for unfamiliar fruit juices. Following pre-tests, twenty-four adults tasted four different juices on twenty, ten, five or zero occasions. Post-tests showed that stated preference was an increasing monotonic function of number of exposures, although the effect was short-lived, declining within days of exposure. Birch \& Marlin (1982) explored the effects of taste exposure on food preferences in 2-3-year-old children who tasted either (a) five novel cheeses on twenty, fifteen, ten, five or two occasions, or (b), in a separate study, five novel fruits on twenty, fifteen, ten, five or zero occasions. Paired choices submitted to Thurstone scaling solutions (Torgerson, 1958) indicated an increase in preference as a function of increasing number of exposures.

Birch et al. (1987) explored the effects of different types of exposure on preferences in 2-, 3- and 5-year-olds. Each child received 'visual exposure' to three foods, taste exposure to another three foods, and no exposure to a final food. Visual exposure entailed requesting the child to look at the food for $10 \mathrm{~s}$ on each trial and, thereby, involved both visual and olfactory input. Taste exposure entailed requesting the child to taste the food and, thus, involved visual, olfactory and taste input. Within each exposure condition (visual or taste) one of the foods was exposed five times, one ten times and one fifteen times over a period of $30 \mathrm{~d}$. Following a paired-choice test, Thurstone Case V scaling solutions (Torgerson, 1958) were positively correlated with exposure frequency. Results showed that taste preferences for foods increased as a function of increasing numbers of exposures and, similarly, visual preferences increased with numbers of sight exposures. Interestingly, foods which had been taste exposed also had enhanced visual ratings while the reverse was not true, i.e. there were no enhanced taste preference judgements made for foods that were visually exposed.

It should be noted, however, that because exposures almost invariably occur in particular social contexts (Rozin \& Kennel, 1983; Rozin, 1984), changing the context may alter effects on preferences (Birch, 1987).

\section{MODELLING}

Exposure to foods often occurs within a social context where others are observed eating or otherwise reacting to particular foods. In a very early study, Duncker (1938) investigated the effects of two different social contexts on food preferences of 2-5-yearolds. Children's preferences shifted (a) after being exposed to peers who exhibited preferences which differed from their own or (b) after they had heard a story featuring a hero who expressed a strong preference for a particular food. Marinho (1942) demonstrated that such social effects were greater and more persistent in the case of foods for which the children did not have a strong initial preference. In a study conducted with children aged 1-2 and 3-4 years by Harper \& Sanders (1975), where each child observed his (or her) mother and a stranger eating a particular food, subjects' eating behaviours 
were more influenced by their mothers than by the strangers, and the changes were greater when the children observed a food being eaten (by mother or stranger) rather than simply having the food presented to them. Birch (1980) studied the effects of in vivo peer modelling on the food preferences of preschool children. Subjects were divided into two groups: 3-4- and 4-5-year-olds. At lunchtime over $4 \mathrm{~d}$ the subjects sat with peers who exhibited different preferences for vegetables from theirs. On day 1 the subject was first offered a choice of vegetables and then saw the peers make their choice. On subsequent days the peers chose first and the subjects chose second. Three measures were used: (1) which foods were chosen, (2) how much of them was consumed, (3) a preference ranking procedure. All three measures showed strong modelling effects with preferences of 3-year-olds being more affected than those of the 4-year-olds. Modelling effects are not restricted, of course, to in vivo observations and may also include video models. These effects may account for the finding that television advertising may influence food preferences in children (Galst \& White, 1976; Reiss, 1977; Goldberg et al. 1978).

\section{REWARDS}

Foods may enter into reward contingencies in at least two ways. First, they may be presented as a consequence or reward for target behaviours. Second, other 'extrinsic' rewards may be presented contingent upon a particular food being eaten. Both kinds of contingent relationship have been investigated experimentally.

Birch et al. (1980) investigated with 3-5-year-old children the effects of presenting foods as rewards. A snack food was presented either (1) as a reward contingent upon any of a number of specified behaviours, or (2) simply paired with adult attention, or (3) in a non-social context (the snack was left in the child's locker), or (4) at snack time. The children showed enhanced preferences for foods presented as rewards or paired with attention; preferences for foods in the other two conditions remained unchanged.

Although this study suggests that presenting foods as rewards for other behaviour enhances preferences for those foods, a very different effect on preference has been reported in studies that have attempted to reward food consumption. For example, in a study by Birch et al. (1982), when 3-4-year-olds consumed particular fruit drinks they were given the opportunity to engage in play activities. The authors reported that being required to drink in order to play had the effect of decreasing preferences (i.e. as indicated by rankings) for the drinks concerned. Similar effects have been reported in a study with 3-5-year-olds by Birch et al. (1984) who used two different rewards (i.e. movie tickets or verbal praise) for drinking flavoured milk. In a study with 4-7-year-olds, Newman \& Taylor (1992) further explored the effects of 'means-end relationships' on food preferences. Two snacks, which initially were equally rated as being moderately preferred, were chosen for study. Children were assigned to a 'means-end' group (where one snack was consumed to gain the other), a temporal-order control group (where one snack was presented and eaten before the other), or a 'mere exposure' control group (where the children chose the order in which they ate the two snacks). Results showed that children in the 'means-end' group came to devalue (i.e. prefer less, as indicated by rankings) the snack used as the means, relative to that used as the reward, while the preferences of children in the other two groups remained approximately equal for both 
snacks. The findings from these studies clearly indicate that rewarding children's consumption of particular foods will not only fail to increase preference but will actually lead to a decrease in preference for those foods. As a consequence of this research, the claim that rewarding food consumption has negative effects on preference has now become widespread in the literature. Our work, described below, questions the generality of this finding.

\section{ENHANCING THE FOOD PREFERENCES OF 5-7-YEAR-OLD CHILDREN AN EXPERIMENTAL STUDY}

This study was designed to investigate the effectiveness of a video-based intervention, which combined both peer modelling and rewards for eating previously-refused foods.

\section{Design}

Much of our research employs single-subject research designs (see Kazdin, 1982). These designs require, for each subject, repeated measures of dependent variables (e.g. amount of target food eaten) before and after the introduction of experimental interventions and, thus, they facilitate analysis of the acquisition, change and maintenance of preferences for specific foods across subjects and time. In the multiplebaseline design used here the introduction of interventions is staggered over time across different subjects and across foods. The design thus controls for amount of preintervention exposure to the foods and for intervention order effects, yielding several replications of independent-variable effects.

\section{Method}

The study was carried out in the subjects' home environment where, to enable observation of behaviour during meal times, concealed video cameras were installed. For each subject six reliably-refused foods (two vegetables, two fruits and two pulses) were identified. Two of these foods (i.e. either two fruits, pulses or vegetables) were presented each day as part of the evening meal in addition to foods normally consumed. The 'additional' foods presented in this study included: celery, broccoli, coleslaw, cauliflower, Brussels sprouts, kiwi fruit (Actinidia chinensis), guava (Psidium guajava), mango (Mangifera indica), lychee (Litchi chinensis), blackeye beans (Vigna catjang), butter beans (Phaseolus lunatus) and chickpeas (Cicer arietinum). Parents were instructed not to present the 'additional' foods at times other than those specified by the experimenters and were trained in the use of an observational scale which they used to estimate the amount of food consumed, in the following categories: approximately 0,25 , 50,75 and $100 \%$.

In the first baseline phase of the experiment (see Fig. 1), each child was presented with a pair of additional foods each evening $5 \mathrm{~d}$ per week, one of which was a 'target' food and the other a 'control' food. (The order of presentation of the three pairs of foods was different across the four children, but remained constant for each child throughout the study.) The duration of this period of exposure differed across children, lasting between 


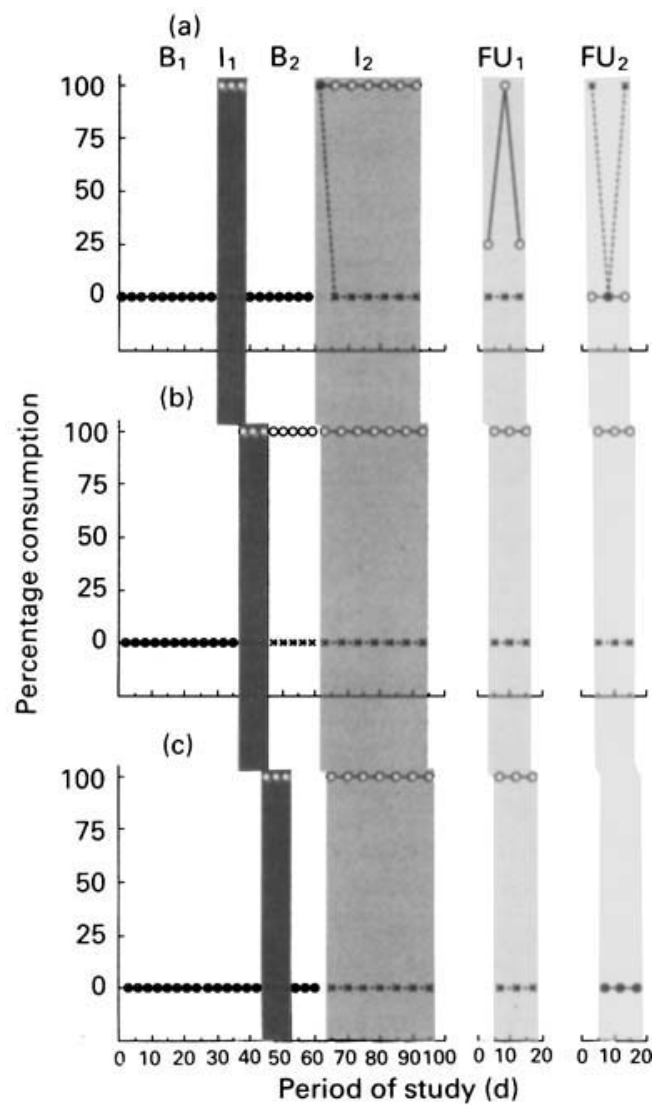

Fig. 1. Percentage consumption, for subject SR, of each of the three pairs of foods, vegetables (a), fruit (b), and pulses $(\mathrm{c})$, when presented at each evening meal. This shows consumption during the first baseline phase $\left(B_{1}\right)$, first intervention phase $\left(I_{1}\right)$, second baseline phase $\left(B_{2}\right)$, second intervention phase $\left(I_{2}\right)$, 2-month follow-up $\left(\mathrm{FU}_{1}\right)$ and 6-month follow-up $\left(\mathrm{FU}_{2}\right)$. (O), Target foods; $(\mathrm{x})$ control foods. (a): (O), celery; $(\mathrm{x})$, coleslaw; (b): (O), kiwi fruit (Actinidia chinensis); (x), lychee (Litchi chinensis); (c): (O), blackeye beans (Vigna catjang); $(\mathrm{x})$, butter beans (Phaseolus lunatus). For details of the experimental procedure and phases of the experiment, see pp. 445-447.

six and ten presentations of each pair of foods, following which the first intervention phase was introduced.

Before the evening meal on day 1 of the first intervention phase, each subject was shown one of a series of short video films that featured a group of 'heroes', older children called the Food Dudes. In each video the Food Dudes ate one of the three target foods (e.g. celery) and, in the context of a 'struggle against the forces of evil', extolled the virtues and enjoyment of eating that food. They also asked the viewer to join the struggle by eating the food which had been featured in the film, which would result also in their getting gifts (e.g. Food Dudes caps and t-shirts) and being able to join the Food Dudes Club. Then during the meal, each subject was presented with the target food featured in the film (e.g. celery), and its control pair member (e.g. broccoli). Reward was contingent 
upon the subject reaching a criterion level of at least $75 \%$ consumption of the target food. Consumption of the control food was also monitored to test for generalization. On subsequent occasions, before watching each video the subject was given a reward if he (or she) had complied with the Food Dudes' request to eat the target food on the previous intervention evening. Each pair of foods was presented in this manner on three separate successive occasions. Baseline conditions (second baseline) were then resumed for at least three further presentations of each pair.

Following this a second intervention phase was introduced. Presentations of each food pair were reduced to one per week over 7 weeks. Subjects were instructed by the Food Dudes to eat the target foods during the 7-week duration of this phase and were told that if they did so they could go on a special outing of their choice. Subjects monitored their own progress by placing a sticker on a monitoring card provided by the Food Dudes, whenever they ate the target food. If by the end of this phase a target of $75 \%$ consumption across the 7 weeks was met, the promised reward was given. Since all children succeeded, tickets for their outings were delivered to all of them within a few days of completing this phase.

Long-term effects of the experimental interventions were investigated in two follow-up phases, after 2-month and after 6-month intervals, during which baseline conditions were reinstated and subjects were presented with each of their three food pairs once weekly for 3 weeks.

\section{Results}

To illustrate the general findings of this study we present, in Fig. 1, detailed results from one of the four subjects, SR. For this subject, the intervention was first introduced for celery, with coleslaw as the control food (Fig. 1(a)), and second for kiwi fruit, with lychee as the control food (Fig. 1(b)), and third for black-eye beans, with butter beans as the control (Fig. 1(c)). Fig. 1 shows that none of the six foods, target or control, was consumed during the first baseline phase. When the first intervention was introduced for each of the three target foods in turn, the child ate all these foods on all three presentations; none of the control food was ever eaten. When the intervention ceased, in the second baseline, consumption of celery and black-eye beans fell to zero but the child continued to eat all the kiwi fruit whenever it was presented.

When the second intervention was introduced the child once again ate all the target foods whenever presented across the 7 weeks of this phase. Apart from the first session, when coleslaw was eaten, there was no evidence of generalization effects to control foods.

At the 2-month follow-up the child ate all the kiwi fruit and the black-eye beans and averaged $50 \%$ consumption of celery. At the 6-month follow-up, while the child still ate all the kiwi fruit, consumption of celery and black-eye beans fell to baseline levels. For reasons that remain to be explained, the control vegetable, coleslaw, was eaten $(100 \%)$ in two of the three sessions of this follow-up.

For all four children both interventions had the effect of increasing their consumption of the target foods to criterion levels on virtually every occasion they were presented during the intervention phases. There was little evidence of generalization of experimental effects to control foods across the different phases of the study. The main difference between individuals occurred in the follow-up phases. While three of the four subjects 
continued to consume the fruits at $100 \%$ levels, there was a decline in consumption of pulses and, particularly, of vegetables although this varied considerably across subjects.

The children's behaviour at evening meals was recorded on video throughout the experiment, including their spontaneous verbalizations and expressions of preferences, likes and dislikes etc. for many of the foods employed. Thus, for example, in the first baseline phase when SR (see Fig. 1) was presented with kiwi fruit distaste was expressed by saying 'I don't like kiwi . . . I hate kiwi, it's really horrible' and pushed the bowl of kiwi fruit away. This is in total contrast to the reactions to this fruit following the introduction of the first intervention, when SR responded by saying, for example, 'These are nice' and by seeking ways of getting extra kiwi fruit from her mother's fruit store. Similar changes in expressed preference were recorded for other children, though only with some of the foods.

\section{Discussion}

The most striking finding of this study was that the interventions resulted in the children eating all targeted foods. These consisted of vegetables, pulses and fruits which the children had consistently refused to eat over many previous weeks. However, having once seen the video presentation for a target food the children would then eat all or almost all that food; this was true thereafter on all ten occasions that that particular food was presented over the 9 weeks of the intervention phases.

It should be noted also that although in the first intervention phase a video was presented in each session and performance was directly rewarded (i.e. by t-shirts, caps etc.), in the second intervention phase this was not the case; videos were not shown and apart from the self-monitoring stickers used by the child, the only reward employed was the promise by the Food Dudes of an outing in the future, when the consumption target had been met. Nevertheless this second intervention was highly effective.

These results indicate that, at least in the age-range 5-7 years, the eating practices of children are extremely malleable. It would appear that under the right conditions children may be influenced to eat a wide variety of fruits, pulses, and vegetables, which previously they have rejected. The generality of these findings, of course, needs to be established with a wider range of foods and with larger numbers of subjects of varying ages and in different cultures.

It is also necessary to identify the specific features of the procedure that had most efficacy in changing food consumption. Repeated sight exposure of the foods in itself had little or no effect on the consumption, which remained mostly unchanged across the first baseline phase.

It is difficult on the basis of this study alone, to assess the relative contributions of peer modelling and of the reward contingencies. There are clear indications, however, that the rewards, or promises of rewards, were a critical ingredient. This is indicated by the abrupt drop-off in consumption of many of the target foods in the second baseline phase and the immediate and sustained recovery of consumption during the second intervention, in which the Food Dudes video was not shown.

The notion that providing rewards contingent upon the consumption of particular foods may lead to enhanced preference of those foods appears to run counter to much of the literature in this area. There was no evidence from any of the children, in relation to any of the target foods, of such a negative effect of reward. Indeed, in the case of some of 
the foods, particularly fruits, consumption was very high 6 months after the end of the second intervention.

This, of course, raises the important question of why rewards in this study were so potent in producing positive behaviour change when they have not had this effect in other studies. One possible explanation concerns the measures used in the different studies. The main outcome measure used in the studies by Birch et al. $(1982,1984)$ and Newman \& Taylor (1992), was verbal statements or rating of preference. Unlike the present experiment, these studies did not focus on what the children did or did not eat. On these grounds it might be argued that providing rewards for eating particular foods, as in our procedure, may have a positive impact on consumption but a negative effect on stated preference. There is, however, no evidence to support this view and, indeed, the spontaneous verbal expressions of preference and other reactions recorded in the course of the study (see p. 448) show, at least for some of the foods, very positive changes in line with actual eating behaviour.

An alternative explanation for the apparent discrepancy in findings between our studies and previous studies concerns the very different contexts in which rewards were employed and, particularly, what they symbolize or convey to the subjects. In the Newman \& Taylor (1992) study, for example, if food A is designated as being the reward for eating food $B$, this may indicate to the child that food $B$ is considered by the experimenter to be less good than food A. If the child is then asked, in a preference test, which is better it is not surprising that food A is selected. Indeed, it is possible to envisage a range of situations when attempts by parents, psychologists and others to directly 'bribe' or persuade children to eat a food may have counter-productive effects, particularly if the reward carries with it the implication that eating the food is not of value in and of itself. This may have been the case in the studies by Birch et al. $(1982,1984)$. The context in which rewards were employed in our study differed markedly. Here the intrinsic virtues and enjoyment of eating the target food were described by the Food Dudes who just happened also to provide additional incentives, by way of the children joining their club and gaining prizes, for success in eating these foods. The 'symbolic' context of reward delivery was, thus, entirely positive and could not easily be construed as indicating that eating the foods was a low-value activity. This suggests that statements about negative (or positive) effects of rewarding food consumption, including preferences, must take into account how the child construes the contingency (Lowe, 1979, 1983; Lowe et al. 1987).

Supporting evidence for the view that rewards presented in an appropriate context should increase the probability of eating the target foods in the future comes from the literature on the effects of taste exposure. If eating a particular food is rewarded then this will mean, by definition, that the food is tasted more often than if no reward had been given. Since a number of studies (Birch \& Marlin, 1982; Pliner, 1982; Birch et al. 1987) have reported that increased taste exposure leads to increased preference, it follows that rewarding food consumption should enhance preference. This may depend, of course, on the number of taste exposures provided and some foods may require different amounts of taste exposure before they acquire intrinsic reinforcing properties. The delivery of 'extrinsic' reinforcers may be very important to ensure that this process takes place.

In the present study only ten tastings of the target foods were rewarded which may have been sufficient to sustain consumptions of the fruits up to the 6-month follow-up in the absence of extrinsic rewards; indeed, as few as three exposures was sufficient to maintain consumption of fruits in most subjects (i.e. in baseline 2). Pulses and vegetables 
may generally require more extensive taste exposure before they become intrinsically reinforcing, although most of the subjects continued to eat some of these items in the follow-up tests. Clearly there are many other factors, including the gradual fading-out of reward, which might enhance the maintenance of changes in eating behaviour and these remain to be investigated in future research.

It is, in addition, important to investigate techniques for promoting generalization of increased consumption to a range of other foods beyond the particular item targeted. The relative contributions of peer influence and of rewards to the success of the present interventions should also be systematically analysed.

A key issue raised by this research, particularly compared with other studies in the literature on food preferences, concerns measures of performance. How is 'food preference' best measured? The possibilities include: (1) a verbal preference or ranking test given immediately before and after a particular intervention, as in most previous research on reward effects, (2) recording of the spontaneous expressions of like, dislike etc. made by subjects when presented with foods during an experiment, (3) recording what people actually do or do not eat, and how much of the food in question they consume. In a number of studies it has been reported that what people say in post-intervention 'tests' often does not correspond with what they have said spontaneously in the course of an experiment (Dugdale \& Lowe, 1990). Disparities between what people say and what they do, in a great variety of contexts, are even better documented (Nisbett \& Wilson, 1977; Baer et al. 1985; Deacon \& Konarski, 1987). In the final analysis the best measure of 'preference' may depend on the particular research objective. If, like that of the present work, it is to understand why people eat what they do and how to alter what they eat, then measures of actual consumption must be paramount. This should also be, of course, the main objective for Government and other agencies concerned with empowering children and adults to eat healthier diets.

\section{ETHICAL CONSIDERATIONS}

The experimental research described was approved by the Department of Psychology Ethics Committee at the University of Wales, Bangor. The parents of the children concerned, who volunteered to have their children participate, were fully consulted on every aspect of the procedure throughout the study. For the children themselves it was an enjoyable experience and it also had the beneficial effect of encouraging them to eat a more varied and healthy diet.

The research reported in this paper was supported by Unilever Research and the Economic and Social Research Council.

\section{REFERENCES}

Baer, J. R., Williams, J. A., Osnes, P. G. \& Stokes, T. F. (1985). Generalized verbal control and correspondence training. Behavior Modification 9, 477-489.

Bentall, R. P. \& Lowe, C. F. (1987). The role of verbal behavior in human learning III: Developmental differences. Journal of the Experimental Analysis of Behavior 47, 177-190.

Bentall, R. P., Lowe, C. F. \& Beasty, A. (1985). The role of verbal behavior in human learning II: Developmental differences. Journal of the Experimental Analysis of Behavior 43, 165-181.

Birch, L. L. (1980). Effects of peer model's food choices and eating behaviors on preschoolers' food preferences. Child Development 51, 489-496. 
Birch, L. L. (1987). Children's food preferences: developmental patterns and environmental influences. Annals of Child Development 4, 171-208.

Birch, L. L., Birch, D., Marlin, D. W. \& Kramer, L. (1982). Effects of instrumental eating on children's food preferences. Appetite 2, 125-134.

Birch, L. L., McPhee, L., Shoba, B. C., Pirok, E. \& Steinberg, L. (1987). What kind of exposure reduces children's food neophobia? Looking vs. tasting. Appetite 9, 171-178.

Birch, L. L. \& Marlin, D. W. (1982). I don't like it; I never tried it: effects of exposure on two-year-old children's food preferences. Appetite 3, 353-360.

Birch, L. L., Marlin, D. W. \& Rotter, J. (1984). Eating as the 'means' activity in a contingency: Effects on young children's food preference. Child Development 55, 431-439.

Birch, L. L., Zimmerman, S. I. \& Hind, H. (1980). The influence of social-affective context on the formation of children's food preferences. Child Development 51, 856-861.

Brown, A. L. \& Campione, J. C. (1984). The faces of transfer: Implications for early competence, individual differences, and instruction. In Advances in Developmental Psychology, vol. 3, pp, 143-192 [M. Lamb, A. Brown and B. Rogoff, editors]. Hillsdale, NJ: Erlbaum.

Catania, A. C. (1992). Learning. Englewood Cliffs, NJ: Prentice Hall.

Catania, A. C., Horne, P. J. \& Lowe, C. F. (1989). Transfer of function across members of an equivalence class. The Analysis of Verbal Behavior 7, 99-110.

Catania, A. C., Lowe, C. F. \& Horne, P. J. (1990). Nonverbal behaviour correlated with the shaped verbal behavior of children. The Analysis of Verbal Behavior 8, 43-57.

Chadwick, P. D. J. \& Lowe, C. F. (1990). Measurement and modification of delusional beliefs. Journal of Consulting and Clinical Psychology 58, 225-232.

Crook, C. K. (1978). Taste perception in the newborn infant. Infant Behavior and Development 1, 52-69.

Davis, R. G. (1978). Increased bitter taste detection thresholds in Yucatan inhabitants related to coffee as a dietary source of niacin. Chemical Senses and Flavour 3, 423-429.

Deacon, J. R. \& Konarski, E. A. Jr (1987). Correspondence training: an example of rule-governed behavior? Journal of Applied Behavior Analysis 20, 391-400.

Desor, J. A., Mallor, O. \& Turner, R. (1973). Taste in acceptance of sugars by human infants. Journal of Comparative and Physiological Psychology 84, 496-501.

Diaz, R. M. (1992). Methodological concerns in the study of private speech. In Private Speech: From Social Interaction to Self-Regulation, pp. 55-81 [R. M. Diaz and L. E. Berk, editors]. Hove, Sussex: Lawrence Erlbaum Associates.

Dugdale, N. A. \& Lowe, C. F. (1990). Naming and stimulus equivalence. In Behaviour Analysis in Theory and Practice. Contributions and Controversies, pp. 115-138 [D. E. Blackman and H. Lejeune, editors]. Hove, Sussex: Lawrence Erlbaum Associates.

Duncker, K. (1938). Experimental modification of children's food preferences through social suggestion. Journal of Abnormal Social Psychology 33, 489-507.

Fieldhouse, P. (1991). Food and Nutrition: Customs and Culture. London: Chapman and Hall.

Fischer, R., Griffin, F., England, S. \& Garn, S. M. (1961). Taste thresholds and food dislikes. Nature 191, 1328.

Galst, J. P. \& White, M. H. (1976). The unhealthy persuader: the reinforcing value of television and children's purchase-influencing attempts at the supermarket. Child Development 47, 1089-1096.

Glanville, E. V. \& Kaplan, A. R. (1965). Food preference and sensitivity of taste for bitter compounds. Nature 205, 851-853.

Goldberg, M. E., Gorn, G. J. \& Gibson, W. (1978). TV messages for snack and breakfast foods: Do they influence children's preferences? Journal of Consumer Research 5, 73-81.

Greene, L., Desor, J. \& Maller, O. (1975). Heredity and experience: their relative importance in the development of taste preference in man. Journal of Comparative and Physiological Psychology 89, 279-284.

Hall, M. J., Bartoshuk, L. M., Cain, W. S. \& Stevens, J. C. (1975). PTC taste blindness and the taste of caffeine. Nature 253, 442-443.

Harper, L. \& Sanders, K. M. (1975). The effect of adults' eating on young children's acceptance of unfamiliar foods. Journal of Experimental Child Psychology 20, 206-214.

Hayes, S. C. (1989). Rule-Governed Behavior: Cognition, Contingencies, and Instructional Control. New York: Plenum Press.

Jefferson, S. C. \& Erdman, A. M. (1970). Taste sensitivity and food aversions of teenagers. Journal of Home Economics 62, 605-608. 
Kazdin, A. E. (1982). Single-Case Research Designs. New York: Oxford University Press.

Levins, R. C. \& Lewontin, R. (1985). The Dialectical Biologist. Cambridge, MA: Harvard University Press.

Lowe, C. F. (1979). Determinants of human operant behavior. In Advances in Analysis of Behavior, vol. 1, pp. 159-192 [M. D. Zeiler and P. Harzem, editors]. Chichester: Wiley.

Lowe, C. F. (1983). Radical behaviorism and human psychology. In Animal: Models of Human Behavior: Conceptual, Evolutionary, and Neurobiological Perspectives, pp. 71-93 [G. C. L. Davey, editor]. New York: Wiley.

Lowe, C. F. (1989). From Conditioning to Consciousness: The Cultural Origins of Mind. Inaugural Lecture. Bangor: University of Wales.

Lowe, C. F., Beasty, A. \& Bentall, R. P. (1983). The role of verbal behavior in human learning: Infant performance on fixed-interval schedules. Journal of the Experimental Analysis of Behavior 39, 157-164.

Lowe, C. F. \& Chadwick, P. D. J. (1990). Verbal control of delusions. Behaviour Therapy 21, 461-479.

Lowe, C. F. \& Higson, P. J. (1981). Self-instructional training and cognitive behaviour modification: a behavioural analysis. In Applications of Conditioning Theory [G. C. L. Davey, editor]. London and New York: Methuen.

Lowe, C. F. \& Higson, P. J. (1983). Is all behavior modification 'cognitive'? In Current Issues in Clinical Psychology, pp. 207-227 [E. Karas, editor]. London: Plenum.

Lowe, C. F. \& Horne, P. J. (1985). On the generality of behavioural principles: Human choice and the matching law. In Behaviour Analysis and Contemporary Psychology, pp. 97-116 [C. F. Lowe, M. Richelle, D. E. Blackman and C. M. Bradshaw, editors]. London: Lawrence Erlbaum Associates.

Lowe, C. F., Horne, P. J. \& Higson, P. J. (1987). The hiatus between theory and practice in clinical psychology. In Theoretical Foundations of Behavior Therapy, pp. 153-165 [H. J. Eysenck and I. Martin, editors]. New York: Plenum.

Marinho, H. (1942). Social influence in the formation of enduring preferences. Journal of Abnormal and Social Psychology 37, 448-468.

Mead, G. H. (1934). Mind, Self and Society. Chicago: University of Chicago Press.

Meichenbaum, D. (1990). Cognitive perspectives on teaching self-regulation. American Journal of Mental Retardation 94, 367-368.

Newman, J. \& Taylor, A. (1992). Effect of a means-end contingency on young children's food preferences. Journal of Experimental Child Psychology 64, 200-216.

Nisbett, R. E. \& Wilson, T. D. (1977). Telling more than we can know: Verbal reports on mental processes. Psychological Review 84, 231-259.

Pliner, P. (1982). The effects of mere exposure on liking for edible substances. Appetite 3, 283-290.

Pressley, M., Borkowski, J. G. \& Schneider, W. (1987). Cognitive strategies: Good strategy users coordinate metacognition and knowledge. In Annals of Child Development, vol. 5, pp. 89-129 [R. Vasta and G. Whitehurst, editors]. Greenwich, CT: JAI Press.

Reiss, E. S. (1977). Advertising and product development. Food Technology 31, 75-77.

Rozin, P. (1982). Human food selection: The interaction of biology, culture and individual experience. In The Psychology of Human Food Selection, pp. 225-254 [L. M. Barker, editor]. Chichester: Wiley.

Rozin, P. (1984). The acquisition of food habits and preferences. In Behavioral Health: A Handbook of Health Enhancement and Disease Prevention, pp. 590-607 [J. D. Matarazzo, J. A. Herd, N. E. Miller and S. M. Weiss, editors]. New York: Wiley.

Rozin, P. \& Kennel, K. (1983). Acquired preferences for piquant foods by chimpanzees. Appetite 4, 69-77.

Simoons, F. J. (1969). Primary adult lactose intolerance and the milking habit: a problem in biological and cultural interrelations I: Review of the medical research. American Jowrnal of Digestive Disorders 14, 819-836.

Simoons, F. J. (1970). Primary adult lactose intolerance and the milking habit: a problem in biological and cultural interrelations. II: A cultural-historical hypothesis. American Journal of Digestive Disorders 15, 695-710.

Torgerson, W. S. (1958). Theory and Methods of Scaling, New York: Wiley.

Vygotsky, L. S. (1987). Thinking and speech. In The Collected Works of L. S. Vygotsky, pp. 39-285 [R. W. Rieber and A. S. Carlton, editors]. New York: Plenum Press (originally published in Russian in 1934).

Whitman, T. L. (1990). Self-regulation and mental retardation. American Journal of Mental Retardation 94, $347-362$.

Woods, P. A. \& Lowe, C. F. (1986). Verbal control of inappropriate social behaviour in mentally handicapped adults. In Science and Service in Mental Retardation, pp. 353-362 [J. Berg, editor]. London: Methuen.

Zajonc, R. (1968). Attitudinal effects of mere exposure. Journal of Personality and Social Psychology 9, 1-27. 